\title{
La importancia del lenguaje en el proceso de reificación de las mujeres
}

\author{
The Importance of Language \\ in the Process of Reification of Women
}

\section{RESUMEN}

Nuestro artículo pretende poner de manifiesto la estrategia de resignificación lingüística puesta en marcha por los patriarcados neoliberales para disimular, dulcificar e invisibilizar todos aquellos significantes que puedan poner en evidencia lo que de facto opera en la prostitución de mujeres y en la gestación subrogada: la reificación de las mujeres. No será la primera vez que nos encontramos ante este ardid. Si la historia ha sido escrita por los vencedores, las palabras con que se escriben no están exentas de violencia epistémica. Para mostrar cómo opera esta estrategia, acudiremos a la reacción antifeminista que se produce en Estados Unidos en la década de los ochenta. Las inversiones lingüísticas puestas en marcha por la nueva derecha contra el movimiento feminista guardan cierto aire de familia con las que operan en el momento actual.

Palabras clave: reacciones patriarcales, gestación subrogada, prostitución, postfeminismo, lenguaje.

\begin{abstract}
Our article aims to highlight the strategy of linguistic resignification implemented by the neo-liberal patriarchates to disguise, sweeten and make invisible all those signifiers that can highlight what de facto operates in the prostitution of women and in surrogacy: reification women's. It will not be the first time that we are faced with this trickery. If the story has been written by the victors, the words with which they are written are not exempt from epistemic violence. To show how this strategy operates, we will turn to the antifeminist resignifications that operate at the present time.
\end{abstract}

Keywords: Backlash, surrogate pregnancy, prostitution, postfeminism, language.

\section{SUMARIO}

1.- Reacción e inversiones semánticas. 2.- La importancia del lenguaje en el proceso de reificación de las mujeres. 3.- Conclusiones. - Referencias bibliográficas. 


\section{Reacción e inversiones semánticas}

La reacción de los años ochenta en Estados Unidos supuso un gran retroceso con respecto a los derechos adquiridos por las mujeres en la década anterior. Esta retracción es especialmente notable en materia reproductiva y legal: reducción de fondos federales para la implantación de políticas de igualdad y para garantizar los derechos reproductivos y sexuales, prohibición del aborto, leyes de protección fetal que vulneraban lo derechos de las mujeres, o el rechazo a ratificar la Enmienda de Igualdad de Derechos.

Las mujeres asistieron a un recorte en sus derechos sociales y en sus posibilidades de promoción a la esfera política, profesional y cultural. El indicador más claro de estar ante una reacción contra las mujeres fue el modo en el que se las culpó de manera individual de su desigualdad. Los problemas de las mujeres dejan de ser problemas sociales, políticos y culturales. El centro de la problemática se desvía a la elección y decisión personal.

Las mujeres han elegido, pero han elegido mal. Lo quieren tener todo, cuando a «todo»-dirá el subtexto- sólo pueden aspirar los hombres. Uno de los grandes legados de la reacción de los ochenta es convencer a las mujeres de que realmente son libres de elegir. La elección se convierte en el argumento fundamental de las tesis liberales, dejando sin resolver dos cuestiones fundamentales, a saber: ¿son buenos todos los fines elegidos libremente? ¿En qué consiste exactamente esa libertad?

Pues bien, en plena reacción, la nueva derecha se impone como tarea la lucha contra el movimiento feminista. La misión es doblemente degradante. Por un lado, en tanto que movimiento reaccionario, se encuentra en situación de dependencia respecto al feminismo. La nueva derecha nunca llevaba la iniciativa, su tarea es de contención, oposición y rechazo. Por otro lado, frente a otros movimientos reaccionarios de la época cuya tarea se presentaba como heroica -pensamos en cómo se percibía la lucha contra el comunismo en plena guerra fría-, la batalla de los neoconservadores es vulgar, intranscendente. Luchar contra mujeres, no tiene nada de heroico (Faludi, 1991).

La táctica utilizada por la nueva derecha será una suerte de resignificación semántica de los términos implicados en el debate en torno a la igualdad. De este modo, su degradante tarea tendría alguna posibilidad de convertirse en una hazaña humanitaria:

Los hombres de la «nueva derecha» llegaron a la conclusión de que, si podían redefinir el significado de los términos en el debate acerca de la igualdad, podrían allanar verbalmente su avance hacia los puestos de mando. Si desbarataban las líneas del poder mediante una especie de inversión semántica, tal vez pudieran dar un golpe maestro utilizando el eufemismo (Faludi, 1991: 355).

Esta táctica no es nueva, guarda un enorme paralelismo con la estrategia utilizada en los años veinte por el Ku Klux Klan, quienes, como recuerda Faludi:

Habían conseguido amplio apoyo utilizando una maniobra retórica semejante, que consistió en minimizar su racismo y presentarlo como patriotismo: no se 
dedicaban a linchar negros, eran reformadores morales que defendían la bandera nacional (Faludi, 1991: 356).

La nueva derecha disimuló su ira contra los derechos adquiridos por las mujeres y presentó su batalla como una defensa de los valores tradicionales y de la familia. Así, rebautizaron como «actitud a favor de la vida» su rechazo a los derechos reproductivos logrados por las mujeres en la última década. Su oposición a la libertad sexual se camufla en una «actitud a favor de la castidad». Su condena a la presencia masiva de las mujeres en el mundo laboral en adelante pasaría a denominarse «actitud a favor de la maternidad».

Las inversiones semánticas llevadas a cabo por la nueva derecha fueron llevadas hasta sus últimas consecuencias. El feminismo se catalogó como un «movimiento contra la familia». Rebautizar al movimiento feminista como «movimiento contra la familia» puede resultar, incluso, galante viendo las derivas que la estrategia acabaría tomando. Las feministas fueron tachadas por los antiabortistas como asesinas de niños, demonios, bárbaras o asesinas. Como ha puesto de manifiesto Susan Faludi, lo que evidencia mejor la naturaleza de esta reacción, son los insultos que proferían a las feministas en voz baja: esos «puta» o «tortillera». Lo que realmente evidencian estos epítetos es que el «mayor pecado de las feministas podría ser muy bien la independencia sexual, más que el asesinato» (Faludi, 1991: 567).

Pues bien, mientras que a las feministas las designan como enemigas de la familia, la nueva derecha definirá su postura regresiva y negativa contra la ampliación de los derechos de la mujer como actitud a favor de la familia (Faludi, 1991: 355). Como vemos, la reacción de la nueva derecha se enmarca sin fisuras dentro de la «Gran Mentira»": no sólo aspiraba a frenar la lucha de las mujeres por la igualdad, pretendía más. En tanto estrategia reactiva perseguía revocar todos sus logros.

George Gilder publica en 1986 Men and marriage; según advierte la portada de la obra, se trata de una versión extendida y revisada de Suicidio sexual (1973). En Men and marriage arremete contra todas las demandas del movimiento feminista alegando a una suerte de reparto natural de las tareas. La inversión de este reparto rompería el equilibrio natural que se encuentra en la base de nuestro desarrollo civilizatorio. Según el autor:

La política ha sido principalmente un dominio masculino en todas las sociedades estudiadas por antropólogos e historiadores. Las mujeres en todas partes, a menos que sean hostigadas por feministas, tienden a dar a los hombres el liderazgo (Gilder, 2008: 109).

Dado que la dominación masculina se encuentra en todas las sociedades civilizadas, feminizar la política supondría violar las tendencias naturales humanas:

El dominio masculino en la política es parte de la constitución sexual de todas las sociedades civilizadas. Esto no significa la imposición de ningún límite a las

2 «Gran Mentira»: cuando más grande es una mentira, más posibilidades hay de que sea creída. 
mujeres extraordinarias que a menudo llegarán a sus propios puestos por sus propios méritos. Pero significa que el esfuerzo de feminizar por la fuerza nuestra aventura viola las profundas propensiones humanas (Gilder, 2008: 109).

Sin duda alguna, la mayor amenaza para los hombres era la libertad reproductiva de las mujeres. Gilder expresó sin contemplación el temor ancestral que los hombres sienten ante este tipo de libertad:

El pene del hombre ya no es un órgano decisivo en sí mismo. Esta demanda feminista de que las mujeres tengan control sobre sus propios cuerpos acentuó el reconocimiento inconsciente de que los hombres han perdido casi completamente el control de la actividad procreadora. Las mujeres son sólo ligeramente dependientes de los hombres -las mujeres pueden concebir un bebé artificialmente o en un solo encuentro. Pero un hombre no puede validar sus poderes reproductivos, su papel en la cadena de la naturaleza, sin la cooperación activa, deliberada y ahora revocable de una mujer. El pene de un hombre se convierte en un juguete vacío a menos que una mujer deliberadamente decida admitir la paternidad de un hombre (Gilder, 2008: 107).

Las resistencias a este cambio en el equilibrio de las relaciones entre hombres y mujeres justifican, a juicio de Gilder, un movimiento conservador que oponga firme resistencia al aborto legal. Como ha sabido ver Faludi (1991: 581), «todas las aspiraciones de las mujeres -en materia de educación, trabajo o cualquier otra forma de autodeterminación- dependen en última instancia de su capacidad de decidir si desean procrear o no y cuándo». Es por esta razón por la que la libertad reproductiva se ha encontrado entre las reivindicaciones prioritarias de los movimientos feministas. No es baladí que sea la que, aún hoy, genere más rechazo.

Los grupos a favor de la vida radicalizaron sus posturas a medida que avanzaba la década de los ochenta. Sus protestas apoyaron su habitual violencia semántica con una desproporcionada violencia física. Así, en las puertas de las clínicas donde se practicaban abortos, se pasó del insulto al homicidio ${ }^{3}$, algo que no deja de ser paradójico en un grupo que se autodenomina a favor de la vida.

El movimiento antiabortista tuvo consecuencias nefastas no sólo para la libertad reproductiva de las mujeres. La constante coacción que ejercieron desencadenó un recorte en las ayudas para centros de control de la natalidad y centros de planificación familiar, con la consiguiente incidencia en la salud sexual y reproductiva de las mujeres. La inversión en investigación farmacológica (anticonceptiva y abortiva) disminuyó considerablemente ante la presión social y política que ejercieron.

3 Según Jon A. Shields (2015) en la década de los noventa, la pérdida de fuerza del «movimiento rescate», frustró a los radicales propensos a la violencia. Inscritos en la organización «ejército de Dios», declararon la guerra: «Nosotros, los remanentes de Dios temerosos de hombres y mujeres de los Estados Unidos de América, declaramos oficialmente la guerra a toda la industria matadora de niños» (Shields, 2015). En 1994, el ministro presbiteriano Paul Jennings Hill, cabecilla del «ejército de Dios», acudió a una clínica de Florida donde asesinó al doctor y sus guardaespaldas. Lejos de ser una anécdota, la lista de atentados perpetrados por estos grupos autodenominados a favor de la vida no ha dejado de crecer desde entonces. 
En su lucha contra los derechos reproductivos, los grupos a favor de la vida generaron una nueva iconografía con consecuencias ontológicas y legales nada despreciables. Las imágenes utilizadas por estos grupos solían mostrar al feto aislado, flotando en un útero, desconectado totalmente de la madre. El feto adquiere todo el protagonismo frente a la madre, mero contenedor. Incomprensiblemente, será el feto el que se eleve a la categoría de sujeto: «El feto es una personita consciente, con una cierta picardía incluso; la madre, un "entorno" pasivo, informe e inanimado. El feto es su ocupante y la madre, su habitáculo transitorio" (Faludi, 1991: 591). Nótense las similitudes discursivas con las posturas que intentan legitimar la gestación subrogada.

Como hemos podido bosquejar, conceder la categoría de sujeto al feto, implicaba proporcionarle derechos legales y sanitarios, reconocimiento que en la práctica se resolvía dando prioridad a los derechos de los fetos sobre los derechos de sus propias madres. A finales de los ochenta, los fetos habían adquirido jurídicamente la categoría de «personas». Aunque en un principio, el reconocimiento de derechos de los fetos se diseñó para protegerles de terceras personas, acabó convirtiéndose en una auténtica campaña de acoso contra las madres:

Una oleada de proyectos de ley sobre «el abandono fetal» inundó las legislaturas estatales. Su propósito era definir como delito las actuaciones de una mujer durante el embarazo que pudieran causar daño al feto por negligencia, entre las cuales figuraban desde no seguir las recomendaciones de su médico hasta ingerir alimentos inadecuados o dar a luz en su casa. Otras iniciativas legislativas pretendían paralizar el consumo de alcohol entre mujeres embarazadas, con condenas de hasta veinticinco años de cárcel para las reincidentes [...] los tribunales llegaron a retirar rutinariamente la «custodia» de sus fetos a las embarazadas con ingresos bajos cuyas prácticas prenatales podrían resultar perjudiciales; luego, después de dar a luz, les quitaban las criaturas, que quedaban bajo la tutela del Estado (Faludi, 1991: 594).

La ampliación de los derechos del feto se hacía restando derechos a la madre. Éstas veían sistemáticamente violado su derecho a la intimidad, siendo sometidas a análisis y pruebas sin su consentimiento para verificar que cumplían con lo que parecía ser su único papel: el de incubadora biológica. Las mujeres fueron reducidas a vasijas vacías, utilizando la descripción que Aristóteles proporcionó en el siglo IV a.C. Las resonancias con el contexto actual se tornan más que evidentes.

\section{La importancia del lenguaje en el proceso de reificación de las mujeres}

En las sociedades igualitarias y en las que aún no lo son, gracias a un giro incompleto y enrevesado de eso que denominamos globalización -incompleto en tanto que no ha sido capaz de extender la igualdad y enrevesado precisamente en tanto que ha sido capaz de exportar las ideas reaccionarias a lugares en donde queda aún mucho por conseguir- la invitación a caer en el discurso postfeminista se vuelve demasiado tentadora. 
Según el postfeminismo en su versión popular ${ }^{4}$, el feminismo ya no es necesario. Vivimos en sociedades igualitarias en las que ya no hay sexismo, por tanto, podemos reivindicar los símbolos y actitudes que antes se consideraban opresivos, pues, sencillamente, ya no pueden leerse en clave sexista (Wlodarczyk, 2010). En el utópico mundo sin sexismo del postfeminismo ya no hay vetos, todo es libertad, elección y diversión. A este tipo de fenómenos pertenece lo que McRobbie (2004: 13) denomina la «irónica normalización de la pornografía».

Pues bien, al auspicio de este espejismo de igualdad surge otro de los mantras de los que se nutre la actual ideología reaccionaria: la libertad de elección. Como ha sabido ver Ana de Miguel (2015), consentimiento y libre elección son los grandes mitos puestos en juego por la alianza neoliberal-patriarcal.

La libertad, en las sociedades neoliberales, se ha transformado en una suerte de competencia o capacidad de los individuos postmodernos: la capacidad de elegir en el mercado. Somos libres en tanto que tenemos capacidad de elegir qué comprar o qué bienes escoger; pero también somos libres de elegir qué vender y qué porciones de nuestro ser queremos introducir en el mercado. Pero en este primer boceto faltan demasiadas variables: sexo, raza, clase, etnia.... Todas estas variables inciden en esa capacidad de compra y venta. Por mucho que el mercado exija el libre consentimiento de las partes, no todas las partes juegan en un plano nivelado.

Sostiene Celia Amorós (2005: 295) que «en el feminismo conceptualizar siempre es politizar. Por eso es tan importante conceptualizar y tratar de conceptualizar bien». Pues bien, uno de los ejes claves a tener en cuenta será, precisamente, cómo operan las reconceptualizaciones reaccionarias. En la reacción actual, en la que confluyen el interés patriarcal y el neoliberal, asistimos a un resurgir creciente de resignificaciones semánticas. El objetivo de esta estrategia es, por un lado, ocultar la obvia reificación de las mujeres, por otra, minimizar la deriva comercial de los pactos metaestables de varones en las sociedades patriarcales neoliberales. Hay dos fenómenos en los que esta estratagema se vuelve claramente visible, a saber, la prostitución de mujeres y la gestación subrogada.

4 Siguiendo a Rosalind Gill (2007) podemos identificar cuatro posibles significados del término «postfeminismo»: (1) como posición teórica y epistemológica, (2) como ruptura histórica con el feminismo de la segunda ola (en este sentido estaría vinculado a la tercera ola), (3) como reacción al feminismo; (4) como «sensibilidad» o «régimen de género» -en palabras de McRobbie (2009). En nuestra propuesta de desambiguación (Ávila, 2017) simplificamos la polisemia del término en dos: postfeminismo en su versión popular y postfeminismo filosófico. El postfeminismo, en su dimensión filosófica, surge de la crítica a la noción «rígida» de identidad que se le atribuye al feminismo de la segunda ola. Autoras como Ann Brooks (1997) lo inscriben en un marco de referencia que abarca la intersección del feminismo con otra serie de movimientos anti-fundacionalitas, incluyendo el postmodernismo, el postestructuralismo y el postcolonialismo. El feminismo en su versión popular surge como un término ideológicamente marcado: es una herramienta ideada por y al servicio de los movimientos neoconservadores. Una de las primeras autoras en utilizar el término "postfeminismo" fue la editora del New York Times, Susan Bolotin. En su artículo «Voices from the post-feminist generation» (1982) da cuenta del cambio generacional y del descrédito que había adquirido el término feminista. 


\subsection{El discurso de la trabajadora sexual}

Escapa a las pretensiones de este artículo realizar una genealogía de los discursos legitimadores del sistema prostitucional ${ }^{5}$, si bien, nos resulta fundamental incidir en el aire de familia que mantienen todos ellos. La nota común a todos estos discursos es omitir la violencia y opresión implícita en todo el sistema prostitucional. Los discursos legitimadores nos ofrecen una imagen mixtificada de la prostitución: una imagen unidimensional en la que sólo podemos ver a las mujeres en situación de prostitución, únicas protagonistas y responsables de todo el entramado. Mediante esta estrategia, se puede trasladar a cada mujer individual el peso de todo el sistema y obviar que la prostitución forzada fue el único destino posible para muchas mujeres. Gracias a este ardid, podemos incidir en la elección individual, obviando que para poder elegir, no basta sólo con estar informadas sobre las desigualdades de poder. Para que un ser humano pueda ejercer su autonomía, para poder elegir, tiene que disponer de opciones.

Otro aspecto a destacar, es la lógica expansiva de los discursos legitimadores. Weininger, último bastión de la misoginia romántica, declaraba su simpatía por la figura de la prostituta a la que comparaba con el conquistador:

Como el conquistador, la prostituta posee el sentimiento de su poder, y no se intimida jamás frente a los hombres, mientras éstos se doblegan frente a ella o frente al hombre superior. Como el gran tribuno, cree que hace la felicidad de todos los individuos con quienes habla (Weininger, 1903: 299).

Esta imagen deformada resuena en los actuales discursos a favor de la prostitución. La resignificación da paso a nuevas mitologías en las que las mujeres en situación de prostitución son suplantadas por mujeres emprendedoras, poderosas, capaces de sacar provecho a lo que Catherine Hakim (2010) llama su «ventaja negociadora». En Capital Erótico. El poder de fascinar a los demás, Hakim arremete contra las feministas e insta a las mujeres a utilizar su «capital erótico» para triunfar. Su tesis de partida es la existencia de un «déficit sexual masculino». Para Hakim (2010: $105)$ este «desequilibrio de interés sexual se vería resuelto por las leyes de la oferta y la demanda como ocurre en otros sectores del ocio». A Hakim no le importa en absoluto si esa demanda de sexo responde a condicionantes sociales o culturales, si sustenta relaciones de poder ni si perpetúa sistemas de dominación. Lo que le interesa a Hakim es mostrar que la demanda de sexo por parte de los hombres proporciona a las mujeres una ventaja negociadora.

La obra es una invitación a que salgamos de nuestras tediosas y aburridas vidas para introducirnos en el mercado de la prostitución, pues, «lo enigmático no es que haya mujeres inteligentes y atractivas dedicadas a la prostitución, sino que no sean más las que lo elijan, por la posibilidad de ganar mucho dinero trabajando relativamente pocas horas» (Hakim, 2010: 163). Las narrativas a favor de la prostitución

5 Utilizamos el término acuñado por Laura Nuño y Ana de Miguel (2017) por la capacidad de reconocer su dimensión calidoscópica y sistémica. 
coinciden en propalar que estamos ante una actividad enormemente ventajosa para las mujeres: el nuevo sector del ocio -nótese el desplazamiento- nos reserva grandes oportunidades.

En esta imagen unidimensional y absolutamente mixtificada, sacamos de plano a los destinatarios y a todo el conjunto de relaciones y pactos que alimentan, sostienen y benefician al sistema. Los hombres quedan exonerados de todo tipo de responsabilidad; la responsabilidad, en última instancia, parece sólo nuestra ${ }^{6}$.

Veamos cómo operan las resignificaciones llevadas a cabo por el lobby de la prostitución para cambiar su imagen y que no recuerde en nada a lo que realmente es, una forma de violencia y opresión. En El ser y la mercancía (2013), Kajsa Ekis Ekman estudia este proceso.

El primer engaño sobre el que nos advierte Ekman es hablar de trabajadora sexual. Pues bien, en estos relatos la trabajadora sexual aparece como una heroína feminista que, por un lado, vendría a resquebrajar «las expectativas anticuadas del comportamiento femenino» (Ekman, 2013: 114); por otro, la trabajadora sexual estaría plenamente informada de las relaciones de poder -transitamos el sendero del postfeminismo.

En el relato de la trabajadora sexual, un relato que se transforma en una suerte de wéstern entre buenos y malos, el lado positivo lo encarnarán las trabajadoras sexuales y tendrán como correlato conceptos como «liberación sexual, el libre albedrío, el derecho al trabajo y el derecho a tomar decisiones sobre el propio cuerpo, así como los derechos de los grupos oprimidos, la homosexualidad, la economía de mercado, el progreso y el comportamiento transgresor» (Ekman, 2013: 113). En el lado negativo de la balanza se situarían feministas y políticos (abolicionistas) a los que se asociarían «características densas y opresivas de la moralidad, la duplicidad, la estigmatización, la hostilidad sexual, el esencialismo, el control estatal, la victimización» (Ekman, 2013: 113-114). En estos relatos no aparece nunca el hombre, su papel, en tanto que representante del patriarcado, es trasladado a las feministas, que serán, en última instancia, las que castigan, censuran y estigmatizan.

Este nuevo relato tiene, al menos, dos consecuencias inmediatas: por un lado, idealizar la prostitución, por otro, demonizar al feminismo y presentarlo como contradictorio. En última instancia, la idealización de la prostitución, o si se quiere, la versión edulcorada planteada por los grupos de presión, acabará planteando serios debates entre las feministas. Ante un relato tal, muchas llegarán a plantearse la posibilidad de la prostitución como actividad emancipatoria. Estamos ante un argumento en el que convergen, de manera inquietante, autores y autoras antifeministas (pensamos en Catherine Hakim), la industria de la explotación sexual, autoras feministas pro-sexo y los discursos misóginos.

El relato sobre la trabajadora sexual omite que la prostitución es «la situación más destructiva en que pueda encontrarse una mujer» (Ekman, 2013: 115). Escapa

6 No es baladí señalar que esta atribución exclusiva de la responsabilidad responde a un modo de proceder misógino. Ya lo decía Weininger (1903: 284), si las mujeres se prostituyen no es culpa de los hombres, «radica en la naturaleza de la mujer; lo que no se es, no se podrá ser». El desplazamiento de lo social (en sentido amplio) a lo personal es una constante. 
a los objetivos de este artículo adentrarnos en los riesgos que la prostitución de mujeres tiene para la salud psicosocial de las mismas, no obstante, conviene recordar que, aunque sean muchos los esfuerzos de resignificación, la realidad manda. Como ha puesto de manifiesto Miguel Llorente (2017), el abuso físico, psicológico, el consumo de sustancias o las enfermedades de transmisión sexual, forman parte del día a día de las mujeres que se encuentran en situación de prostitución.

El hombre, decíamos, es el gran ausente en estos discursos. La prostitución, hasta nuestros días, es un «asunto de mujeres». Como ha puesto de manifiesto Beatriz Ranea (2017: 135): «la prostitución tiende a identificarse únicamente con las mujeres en prostitución. En el imaginario colectivo prostitución es sinónimo de prostituta, como si la prostituta encarnase en sí misma una institución tan compleja». El hombre, el demandante, el consumidor y usuario, es el que hace posible la prostitución y siempre ha permanecido invisible (Salazar, 2017; De Miguel, 2015, Gómez Suárez, Pérez Freire y Verdugo, 2015). El nuevo relato de la trabajadora sexual, correlata con el relato del cliente que asegura que la identidad colectiva e individual de los puteros y la generización de la prostitución, permanezcan ocultas ${ }^{7}$. Esta estrategia invisibiliza que nos movemos en un terreno de profundas desigualdades, opresión y violencia. María José Guerra (2017), Laura Nuño y Ana de Miguel (2017), insisten en la resignificación que se produce en el lenguaje para enmascarar un fenómeno generizado y globalizado. Siguiendo las aportaciones de Guerra:

La deriva neoliberal impone hablar de «clientes», empresarios de la industria del sexo y de provisión de servicios, pero justo esta pretendida asepsia mercantil es la que se levanta ante nosotros para enmascarar la opresión ejercida por un sistema prostitucional ahora globalizado y mediado por la movilidad de sus agentes, ya sea la de la «demanda» ya sea la de la «oferta» (Guerra, 2017: 2).

En esta línea, el interés por marcar el carácter aséptico de lo que supuestamente son «meras relaciones comerciales», es reforzado por el discurso que incide en hablar de clientes en lugar de puteros o prostituidores. El discurso del «cliente» introduce otro nuevo giro de opresión característico de la nueva alianza neoliberalismo y patriarcado. Bajo este discurso la relación de poder será doblemente marcada: por un lado, la incorporación del lema (neo)liberal «el cliente siempre tiene razón» -donde el cliente es siempre un hombre. Por otro, la patriarcal disponibilidad de las mujeres para satisfacer los deseos de los hombres, en este caso, de los hombres dispuestos a pagar.

En el nuevo discurso de la trabajadora sexual ya no se vende «el cuerpo», el cuerpo es algo demasiado personal, lo que se vende es un servicio sexual (Ekman, 2013). Esta resignificación es de vital importancia en tanto que nos traslada a un nivel cada vez más elevado de abstracción. En el primer nivel de abstracción se produce una asimilación-escisión entre yo/cuerpo: no se vende el «yo», se venden «cuerpos». En el segundo nivel, se produce una asimilación-escisión entre cuerpo/

7 El 99,7\% de las personas que demandan prostitución son hombres (Gómez Suárez, Pérez Freire y Verdugo, 2017). 
sexo, de tal manera que tampoco es el cuerpo lo que se vende, lo que se vende es «sexo». En el tercer nivel de abstracción, el sexo acaba reducido a un servicio. $\mathrm{Y}$ en tanto que servicio "puede convertirse en divisas, intercambiables en el mercado y, por tanto, socialmente aceptado» (Ekman, 2013: 133).

\subsection{Madre ¿no hay más que una?}

Los intentos del mercado por legitimar la mercantilización de bebés y el alquiler de madres nos permiten dar cuenta de la exuberante creatividad en materia de resignificación conceptual. En primer lugar, la mercantilización de bebés y el alquiler de madres, por muy sólida que sea la alianza entre el neoliberalismo y el patriarcado, resulta difícil de legitimar. Como hemos apuntado, la prostitución cuenta con siglos de legitimación, la tarea reaccionaria consiste en una suerte de modernización del prolífico argumentario misógino y patriarcal, dándole un giro progresista e incluso, como hemos podido señalar, feminista. Es, si se quiere, un intento de disimular la caspa que subyace al sistema con pequeños toques de brillantina.

La gestación subrogada requiere un esfuerzo de resignificación extra para conseguir naturalizar un doble atentado contra los derechos humanos, a saber, construir todo un argumentario para que parezca que los únicos derechos humanos son los del consumidor-comprador y, de este modo, legitimar lo, a priori, ilegítimo.

Lo primero que observamos cuando nos acercamos a los discursos a favor de la gestación subrogada es que el término «madre» desaparece. Como pone de manifiesto Ekman (2013: 196) la madre biológica «es llamada portadora, proveedora, maleta, incubadora, madre sustituta». La investigadora analiza la definición de «madre» que aparece en el Oxford English Dictionary «progenitora de un ser humano; una mujer en relación con el hijo o los hijos a los que dio a luz». Si realizamos la misma consulta en el Diccionario de la Real Academia Española (2017) el resultado es similar en tres de las acepciones reconocidas: «1. $\mathrm{f}$. Mujer o animal hembra que ha parido a otro de su misma especie. 2. f. Mujer o animal hembra que ha concebido. [...] 6. f. Matriz en que se desarrolla el feto».

Desde el punto de vista etimológico se incurre en un error al utilizar el término subrogación. La definición de «sustituto» es «remplazo». Si nos mantenemos fieles a la definición, es a la mujer que paga a la que habría que llamar sustituta, pues es la compradora la que sustituye a la madre que da a luz. Pero las palabras elegidas para construir la(s) narrativa(s) dan cuenta de quién ostenta el poder, «la madre verdadera es la que tiene recursos económicos, mientras que la madre falsa solo tiene su propio cuerpo» (Ekman, 2013: 196).

Mediante esta estrategia, desaparece la vinculación emocional a la que nos traslada el uso del término «madre». Hablar de sustitutas, de portadoras o ignorar por completo a la madre biológica recurriendo a metonimias con la fecundación in vitro, permite asimilar la maternidad subrogada a una técnica de fecundación.

Otra de las estrategias utilizadas en los discursos a favor de la gestación subrogada, es apelar a las familias felices, que no son otra cosa que familias bien posicionadas socialmente, con muy buenos recursos económicos y que, por ende, 
no dudarán en facilitar todo lo necesario para el desarrollo óptimo de las menores y los menores. En este tipo de discursos, la madre gestante será también una «madre feliz» excluida, eso sí, de la felicidad familiar. El relato nos conduce a una suerte de mundo mágico de ilusión, ponis y arcoíris, en el que no queda rastro de transacciones económicas, madres biológicas sometidas a contratos de esclavitud (Nuño, 2016) o la mercantilización de bebés.

Desde los medios de comunicación de masas se promueve una imagen positiva de la gestación subrogada. El poder de los medios de comunicación para introducir cambios significativos en la opinión pública es incuestionable y, en este caso, el cambio juega en contra de las mujeres. La ideología de la mercantilización del capital reproductivo busca legitimarse a toda prisa, la campaña de educación a la ciudadanía ha comenzado. Tal vez la larga lista de famosos -futbolistas, actores, cantantes, empresarios, tertulianos- que hacen proselitismo de la gestación no es suficiente, ofrecen una imagen alejada y esnob del asunto. Por eso hay que intentar ofrecer un rostro cercano, humano, emocional, accesible a cualquiera ${ }^{8}$.

Asistimos al nacimiento de un nuevo mito, el del «varón procreador», que vendría a cumplir con un «elemento central de la ideología patriarcal» (Nuño, 2016), a saber, la sacralización de la genética. Los varones ${ }^{9}$ - con dinero- pueden cumplir el viejo sueño patriarcal de transmitir su carga genética asegurándose la propiedad absoluta de su descendencia. Por decirlo con Lina Gálvez, «el progenitor donante se garantiza la paternidad biológica algo que históricamente ha sido importante para los varones como garantía de dar continuidad a su estirpe y frente a la incertidumbre de su paternidad real» (Gálvez, 11 de diciembre de 2016).

\section{Conclusiones}

La mercantilización de los cuerpos de las mujeres ${ }^{10}$-ya sea con fines reproductivos o con fines sexuales- dinamita cualquier pretensión de construir una sociedad igualitaria. ¿Qué sociedad están construyendo los hombres que demandan dichos servicios? Por decirlo con Ana de Miguel:

8 El programa televisivo «Héroes, más allá del deber» (Cuatro, 19 de octubre de 2010), expone la rutina diaria de cuatro «héroes» y una «heroína»: un policía, un médico de urgencias, un médico del 061, un bombero y una guardia civil. Desde un enfoque humano, acompañamos a los protagonistas en su vida profesional y en su vida privada. Julio Armas, un entregado médico de urgencias, dedicado, cariñoso, hijo y hermano ideal, consigue que espectadoras y espectadores establezcan un vínculo emocional con él. La visita de su madre, que habita junto a toda su familia a 6.000 kilómetros de distancia, nos permite trascender a una dimensión íntima. Julio, separado de su mujer tras asumir su homosexualidad, ha decidido «tener» un bebé por gestación subrogada. Pues bien, ¿quién va a poner en duda los «legítimos» deseos de este héroe? ¿Quién enseña la parte oculta de todo este entramado de mercantilización de seres humanos?

9 Las mujeres también. El mercado de los vientres de alquiler está destinado tanto a parejas (homosexuales y heterosexuales) como a personas solas que deseen tener hijos e hijas. Como ha puesto de manifiesto Lina Gálvez (2016): «Los videos promocionales se centran en las parejas demandantes, normalmente blancas y heterosexuales, aunque las estadísticas nos digan que a esta práctica acceden principalmente parejas de hombres homosexuales».

10 Es importante no olvidar que no hay cuerpo sin sujeta. 
Un mundo más injusto en el sentido fuerte de la palabra. Un mundo en el que cada día tiene menos sentido la máxima kantiana de que las personas no son medios, sino fines en sí mismas. La prostitución de mujeres es para los hombres una escuela de egolatría, prepotencia y negación de toda empatía, en la que priman sus deseos y no importa en absoluto lo que vivan y sientan las mujeres prostituidas. Es una auténtica escuela para aprender e interiorizar las relaciones de desigualdad (De Miguel, 2015: 178).

La prostitución de mujeres y la gestación subrogada, grandes bastiones de la ideología patriarcal, requieren una respuesta clara por parte de aquellas y aquellos que luchan por la construcción de una sociedad igualitaria. Sin embargo, los mitos reaccionarios de la libre elección y el emprendimiento sexual -el empoderamiento que proporciona la venta del capital erótico y/o reproductivo-, la ingenuidad voluntarista de las "prácticas subversivas»" ${ }^{11}$ o el giro neoliberal a los eslóganes radicales -«lo personal es político» y «mi cuerpo es mío»-, se cuelan, acríticamente, gracias al entramado de resignificaciones lingüísticas que hemos analizado.

Los discursos antifeministas se nutren del poder de conceptualización del feminismo para resignificarlos, invertirlos y vaciarlos. Esta estrategia se torna evidente cuando topamos con los derechos sexuales y reproductivos de las mujeres. Hemos analizado los dos escenarios en los que las resignificaciones lingüísticas han sido más fructíferas y evidentes, a saber, en la reacción de los ochenta y en las actuales posturas a favor de la prostitución y a favor de la gestación subrogada.

En la década de los ochenta, los discursos antifeministas puestos en marcha por la nueva derecha contra los derechos reproductivos adquiridos por las mujeres hacen alarde de una ingeniosa capacidad de resignificación. Apelar a los valores

11 ¿La etiqueta comercial afecta al resultado de la acción? ¿Subvierte quien quiere o quién puede? ¿Hasta dónde pueden ser los feminismos resignificados sin incurrir en contradicción? Goldman, Heath y Smith (1991: 336) consideran que «las revistas femeninas intentan redefinir el feminismo a través de las mercancías, interpretando las relaciones cotidianas de las mujeres y negociando una serie de "actitudes" que luego pueden "usar"». En este contexto, el feminismo sería reducido a un mero significado que podría ser recodificado por los publicistas como una secuencia de significantes cosificados. De este modo, el «feminismo comercial» elimina uno de los ejes vertebradores de los feminismos: la dimensión de denuncia social. El feminismo comercial se despolitiza y se transforma en una forma de vida individual. Una vez que se ha eliminado su dimensión social y política, lo que queda es un discurso individual en el que el mito de la libre elección opera como un paliativo de todos los condicionantes sociales, económicos y políticos. Bajo esta deriva popular del feminismo, el término queda rebajado a una suerte de etiqueta comercial, un adjetivo con una suerte de halo redentor que puede ser anexado a determinadas mercancías o prácticas para eximir de cualquier acusación de sexismo. En este sentido nos parece especialmente ilustrador el uso del concepto pornografía feminista. «¿Es acaso el feminismo un adjetivo o una etiqueta para ocupar un segmento del mercado?», preguntaban Ana de Miguel y Laura Favaro (2016). Su respuesta es contundente: «El feminismo no es un adjetivo para hacerse hueco en el mercado ni para tergiversar y vaciar una tradición teórica y política, sino un movimiento social que ha emprendido una lucha tenaz contra un mundo injusto. El feminismo es una teoría crítica del poder y de sus consecuencias para todos los seres humanos; para el propio concepto de lo que es una persona y lo que se puede hacer con ella. Analiza las formas de producción de subjetividades y presenta proyectos alternativos al «esto es así y así lo tienes que aceptar». Presenta proyectos colectivos más allá del «a mí me gusta» o «a mí me pone» o «hay tantos feminismos como mujeres, millones» (De Miguel y Favaro, 2016). 
tradicionales y a la familia les permite ocultar que se trata de un movimiento profundamente reactivo. El feminismo se llevó la peor parte en los procesos de resignificación. Fue catalogado como movimiento contra la familia. Las feministas, en adelante, serían tachadas por los antiabortistas como asesinas de niños, demonios, bárbaras o asesinas. Otro tipo de insultos comunes como puta o tortillera evidencian la aversión, también recordada por Faludi, a la autonomía sexual de las mujeres.

Como hemos estudiado, en el escenario actual los procesos de conceptualización antifeministas estarían íntimamente ligados con los procesos de reificación de las mujeres. El intento de legitimación de la prostitución de mujeres y de la gestación subrogada son los grandes campos de batalla en los que operan las actuales estrategias de resignificación. La lógica del neoliberalismo pretende tratarnos -en

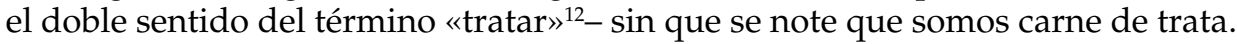

\section{REFERENCIAS BIBLIOGRÁFICAS}

Amorós, Celia (2005). La gran diferencia y sus pequeñas consecuencias... para las luchas de las mujeres, Madrid: Cátedra.

Amorós, Celia (1992). «Notas para una teoría nominalista del patriarcado» en Asparkía. Investigació feminista, Nº 1 , pp. 41-58.

Ávila, María (2017). «Crónica de un matricidio anunciado» en Revista Femeris, Vol. 2, No2, pp.184-202.

Bolotin, Susan (1982). «Voices from the post-feminist generation» en The New York Times Book Review. Disponible en: http:/ /www.nytimes.com/1982/10/17/ magazine/voices-from-the-post-feminist-generation.html?pagewanted=all (Fecha de consulta 25/02/18).

BrooKs, Ann (1997). Postfeminisms: Feminism, cultural theory and cultural forms, New York: Routledge.

CuAtro (2010). "Julio DA LA noticia a su madre: "El año que viene, quiero tener un hijo"» en Cuatro, Disponible en: http://www.cuatro.com/ heroesmasalladeldeber/Julio-noticia-madre-viene-quiero_2_2453355143.html (Fecha de consulta 27/12/17).

De Miguel, Ana y Laura Favaro (2016). «¿Pornografía feminista, pornografía antirracista y pornografía antiglobalización? Para una crítica del proceso de pornificación cultural» EN Labrys, études féministes/ estudos feministas, N 29.

De Miguel, Ana (2015). Neoliberalismo sexual. El mito de la libre elección, Madrid: Cátedra.

EKMAN, Kajsa Ekis (2013). El ser y la mercancía. Prostitución, vientres de alquiler y disociación, Barcelona: Edicions Bellaterra, 2017.

FALUdi, Susan (1991). Reacción. La guerra no declarada contra la mujer moderna, Barcelona: Círculo de lectores, 1993.

FAVARo, Laura (2017). Transnational technologies of gender and mediated intimacy, Unpublished thesis, Londres: University of London.

12 Manejar algo y usarlo materialmente. Proceder con una persona de determinada manera, de obra o de palabra. (Real Academia Española, 2017). 
GÁlvez, Lina (2016). «Vientres de alquiler o la mercantilización de la vida» en Diario.es. Disponible en: http://www.eldiario.es/andalucia/desdeelsur/ Vientres-alquiler-mercantilizacion-vida_6_589751029.html (Fecha de consulta 27/12/17).

GILDER, George (1973). Suicidio sexual, Barcelona: Grijalbo, 1976.

Gilder, George (2008). Men and Marriage. Gretna: Pelican Publishing Company.

Gill, Rosalind (2007). Gender and the Media, Cambridge: Polity Press.

Goldman, Robert; Heath, Deborah \& Sharon L., Smith, (1991). "Commodity

Feminism» en Critical Studies in Mass Communication, No8, pp. 333-351.

Gómez Suárez, Águeda; Pérez Freire, Silvia y Rosa María Verdugo (2015). El putero español. Quiénes son y qué buscan los clientes de prostitución, Madrid: Catarata.

GuerrA, María José (2017). «Apunte sobre geopolítica de la prostitución. Escalas, localizaciones y factor migratorio» en NuÑo, Laura y Ana DE Miguel (dirs.) (2017). Elementos para una teoría crítica del sistema prostitucional, Granada: Editorial Comares, pp. 1-17.

HАкім, Catherine (2010). Capital erótico. El poder de fascinar a los demás, Barcelona: Debate, 2017.

LLORENTE, Miguel (2017). «Impacto de la trata y la prostitución sobre la salud de las mujeres» en Nuño, Laura y Ana De Miguel (dirs.) (2017). Elementos para una teoría crítica del sistema prostitucional, Granada: Editorial Comares, pp. 77-87.

MackInnon, Catharine A. (1989). Hacia una Teoría Feminista del Estado, Madrid: Cátedra, 1995.

MackInnon, Catharine A. y Andrea Dworkin (eds.) (1997). In Harm's Way, The Pornography Civil Rights Hearings, Cambridge: Harvard University Press.

McRoвbie, Angela (2004). «Notes on postfeminism and popular culture. Bridget Jones and the new gender regime» en HARris, Anita (ed.) (2004). All about the Girl. Culture, Power, and Identity, New York: Routledge, pp. 3-14.

McRoвbie, Angela (2009). The Aftermath of Feminism: Gender, Culture and Social Change. London: Sage.

NuÑo, Laura (2016). «Una nueva cláusula del Contrato Sexual: vientres de alquiler» en Isegoría, N55, pp. 683-700. doi: 10.3989/isegoria.2016.055.15

Nuño, Laura y Ana De Miguel (2017). «Presentación», en Nuño, Laura y Ana De Miguel (dirs.) (2017). Elementos para una teoría crítica del sistema prostitucional, Granada: Editorial Comares, pp. VII-XIII.

RANEA, Beatriz (2017). "(Re)pensar la prostitución desde el análisis crítico de la masculinidad» en Nuño, Laura y Ana De Miguel (dirs.) (2017). Elementos para una teoría crítica del sistema prostitucional, Granada: Editorial Comares, pp. 135-142.

SAlAzAR, Octavio (2017). «Prostitución y desigualdad: la necesaria deslegitimación de los sujetos prostituyentes» en NuÑo, Laura y Ana De Miguel (dirs.) (2017). Elementos para una teoría crítica del sistema prostitucional, Granada: Editorial Comares, pp.157-168.

SHIELDS, Jon A. (2015). «Despite planned parenthood shooting, abortion-related violence used to be worse» en The Washington post. Disponible en: https: / / www. 
washingtonpost.com/news/acts-of-faith/wp/2015/11/30/why-the-plannedparenthood-shooting-could-actually-reflect-a-historical-decline-in-abortionrelated-violence/?utm_term $=.230 \mathrm{~b} 7 \mathrm{ec} 01707$ (Fecha de consulta 21/03/18).

WeININGer, Otto (1903). Sexo y carácter, Buenos Aires: Losada, 1952, $3^{\mathrm{a}}$ ed.

WlodarczYK, Justyna (2010). Ungrateful daughters: third wave feminist writings, Newcastle: Cambridge Scholars Publishing.

Recibido el 31 de marzo de 2018 Aceptado el 2 de septiembre de 2018 BIBLID [1132-8231 (2018): 101-115] 